\title{
Tissue-resident memory $T$ cells live off the fat of the land
}

\author{
Cell Research (2017) 27:847-848. doi:10.1038/cr.2017.49; published online 31 March 2017
}

\begin{abstract}
The consumption of exogenous free fatty acids by tissue-resident memory $T\left(T_{r m}\right)$ cells is critical for their longterm survival and antiviral function, and appears to be a conserved feature of $T_{r m}$ cells in both mouse and man, a recent paper published in Nature demonstrates.
\end{abstract}

The generation and long-term survival of pathogen-specific $T$ lymphocytes that are quantitatively and qualitatively advantaged for accelerated pathogen clearance upon re-infection is a defining feature of immunological memory. Historically categorized by their ability to recirculate between tissues or lymphatics by way of blood [1], the recent identification of pathogen-specific memory $T$ cells permanently parked within host tissues has re-defined our concept of memory $\mathrm{T}$ cell immunosurveillance [2, 3]. Such tissue-resident memory $T\left(T_{\mathrm{rm}}\right)$ cells are abundant within barrier tissues including the urogenital tract, intestine and skin, and are critical for the rapid interception of pathogens which commonly breach these sites. However, unlike recirculating memory $\mathrm{T}$ cells whose homeostatic maintenance requirements have been well-defined, the signals supporting $\mathrm{T}_{\mathrm{rm}}$ cell longevity and function within barrier tissues remains largely ambiguous.

In a recent paper published in Nature, Pan et al. [4] demonstrate that $\mathrm{T}_{\mathrm{rm}}$ cells within the skin, a lipid-rich but nutrientpoor microenvironment, exploit exogenous free fatty acid (FFA) metabolism for their long-term survival and antiviral function. In contrast to recirculating memory $\mathrm{T}$ cells, transcriptional profiling revealed skin $T_{\mathrm{rm}}$ cells are induced to strongly upregulate genes facilitat- ing exogenous FFA acquisition and metabolism including Fabp4, Fabp5, Ppary, and the lipid-scavenging receptor $C d 36$. When circulating and skin $\mathrm{T}_{\mathrm{rm}}$ cells were incubated with exogenous fluorescently-conjugated FFAs, only the latter strongly incorporated the probe. Therefore, skin $T_{\mathrm{rm}}$ cells readily acquire exogenous FFAs while their circulating counterparts do not. Upon deletion of the lipid transport molecules FABP4 and FABP5 (herein referred to as Fabp4/5 DKO), skin $T_{r m}$ cells lost their ability to incorporate fluorescently-conjugated FFAs, demonstrating that these transporters are paramount for the internalization of exogenous lipids. To test the relevance of exogenous FFA acquisition in vivo, wild-type and Fabp 4/5 DKO $\mathrm{CD}^{+} \mathrm{T}$ cells were co-transferred into virally infected recipients and memory formation was tracked over the course of three months. While both genotypes formed stable and numerically equivalent populations of circulating memory T cells, skin $\mathrm{T}_{\text {rm }}$ cells of Fabp 4/5 DKO origin began to precipitously wane after one month. This attrition was shown to be the result of increased cell death and not tissue re-localization, suggesting that loss of exogenous FFA acquisition impairs skin $\mathrm{T}_{\mathrm{rm}}$ cell longevity.

Nutrient availability and metabolic programing dictate $\mathrm{T}$ cell function and fate. Circulating naive T cells with limited biosynthesis achieve their modest metabolic demands through glucose catabolism and oxidative phosphorylation (OXPHOS), the mitochondrial generation of ATP from biomolecule oxidation [5]. Upon TCR engagement the cellular metabolic state switches from catabolism to anabolism to en- able rapid cell division, highlighted by rapacious nutrient uptake supporting the synthesis of new lipids, proteins and nucleic acids which will eventually comprise daughter cells. Contraction and memory formation sees a return to metabolic quiescence dominated by OXPHOS [6]. However, unlike naive $\mathrm{T}$ cells reliant on glucose, circulating memory $\mathrm{T}$ cells utilize endogenously synthesized fatty acids for OXPHOS $[7,8]$. The metabolic substrates used by $\mathrm{T}_{\mathrm{rm}}$ cells for OXPHOS were previously unknown. To investigate whether $T_{r m}$ cells similarly employ endogenously synthesized fatty acids for OXPHOS or instead metabolize exogenous FFAs as an energy source, Pan et al. performed fatty acid oxidation experiments using the Seahorse platform. Briefly, oxygen consumption rate (OCR) reflects mitochondrial $\beta$-oxidation (OXPHOS) of experimentally supplemented fatty acids. Pan et al. observed that $\mathrm{T}_{\mathrm{rm}}$ cells rapidly increased OCR following exogenous FFA provision in a manner dependent on FABP4/5 whereas OCR was not changed in circulating memory $\mathrm{T}$ cells when they were provided with exogenous FFAs. These data reveal that homeostatic metabolism varies considerably between memory $\mathrm{T}$ cell populations with differential migratory properties (Figure 1).

The authors next sought to examine the role of exogenous FFA metabolism in $T_{\mathrm{rm}}$ cell-intrinsic antiviral immunity. However, the direct interpretation of $\mathrm{T}_{\mathrm{rm}}$ cell antiviral function is confounded by their ability to potently recruit and synergize with circulating $T$ cells to establish a protective microenvironment $[9,10]$. FTY720, a S1PR1 antagonist, 


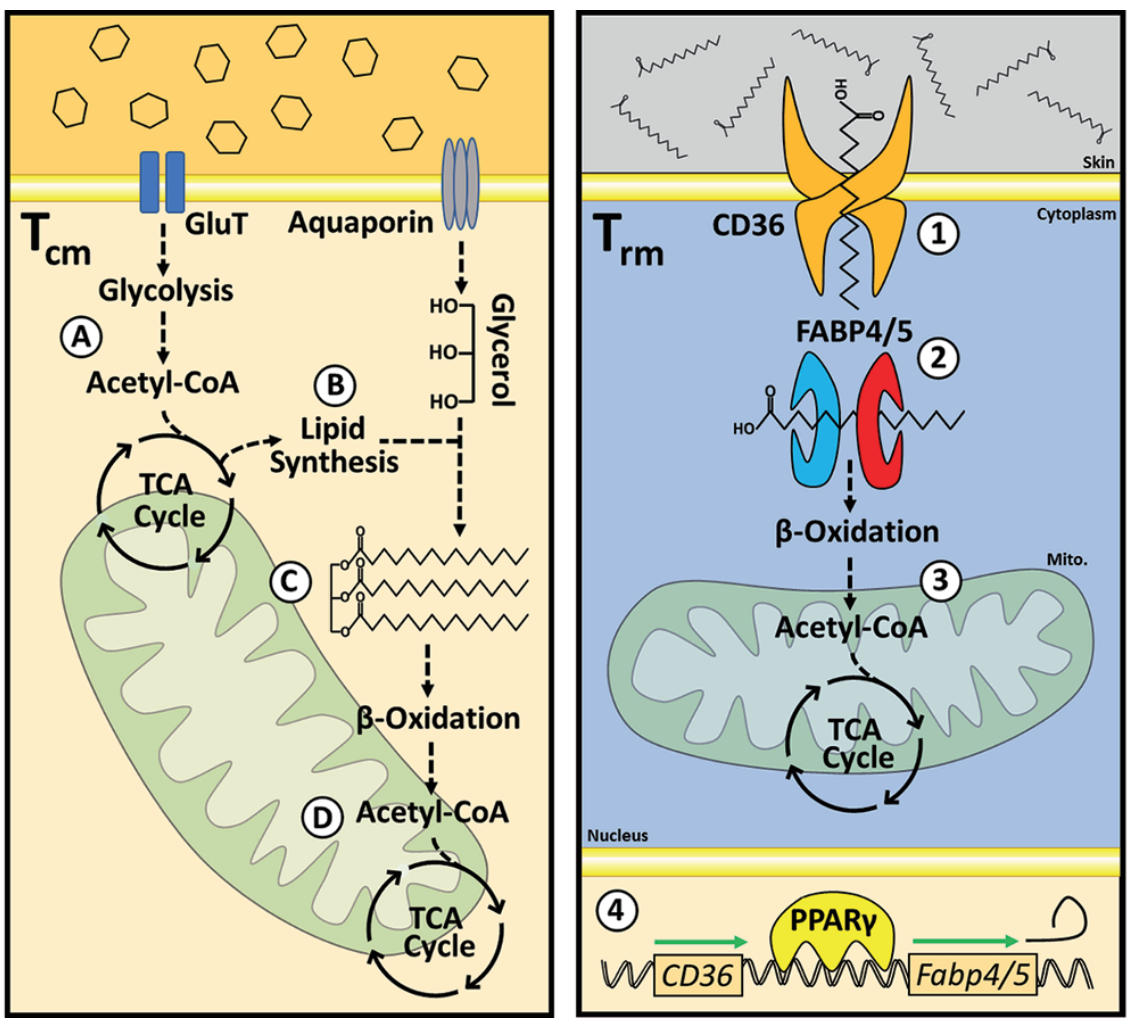

Figure 1 Left, Endogenous lipid metabolism in circulating memory T cells. (A) Basal glucose uptake and glycolysis drives TCA cycle progression. (B) TCA cycle intermediates are utilized for de novo lipid synthesis. (C) IL-7 signaling supports aquaporin-mediated import of glycerol used in the synthesis of triacylglycerol. (D) Triacylglycerol is the predominant substrate for mitochondrial $\beta$-oxidation driving OXPHOS in circulating memory $T$ cells. Right, Exogenous fatty acid utilization by $T_{r m}$ cells. (1) Expression of the lipid scavenger receptor CD36 encourages the uptake of exogenous FFAs. (2) FABP4/5 are necessary for the internalization and processing of exogenous FFAs used for OXPHOS (3). (4) Constitutive expression of Ppary supports exogenous lipid metabolism in $\mathrm{T}_{\mathrm{rm}}$ cells in part through promoting Fabp4/5 expression.

is commonly used to inhibit $\mathrm{T}$ cell recruitment into tissues by blocking their egress from secondary lymphoid organs. Therefore, mice with previously established wild-type or Fabp4/5 DKO skin $\mathrm{T}_{\mathrm{rm}}$ were treated with FTY720 and contemporaneously re-challenged with virus on day 25 , a timepoint earlier shown to precede Fabp4/5 DKO skin $\mathrm{T}_{\mathrm{rm}}$ cell survival defects. With this blockade, Fabp4/5 DKO T $\mathrm{T}_{\mathrm{rm}}$ cells displayed a pronounced impairment in viral clearance revealing a $T_{\mathrm{rm}}$-intrinsic antiviral defect in the absence of exogenous FFA metabolism.
Relating these studies to human health, FABP4 and FABP5 were shown to be uniquely expressed by $\mathrm{CD} 8^{+} \mathrm{T}$ cells in human skin and enriched in human psoriatic lesions where they coexpressed the canonical $\mathrm{T}_{\mathrm{rm}}$ cell marker CD69. Consistent with mouse studies, $\mathrm{CD}^{+} \mathrm{T}$ cells isolated from human skin readily acquired fluorescently-labeled FFAs in ex vivo cultures, while circulating $\mathrm{T}$ cells did not. Taken together, the study presented by Pan et al. strongly suggests that the acquisition and metabolism of exogenous FFAs by skin $\mathrm{T}_{\mathrm{rm}}$ cells represents a necessary and conserved feature of mammalian barrier immunosurveillance. It is often said "you are what you eat". Considering the study by Pan et al., it will now be important to determine how location-specific $\mathrm{T}$ cell diets impact their differentiation and function. Indeed, $\mathrm{T}_{\mathrm{rm}}$ cells vary in their reliance on IL-15 for survival depending on the anatomic location that they inhabit $[11,12]$; further work is needed to determine whether $\mathrm{T}_{\mathrm{rm}}$ cells' dependence on exogenous FFAs is universal or tissue-specific. Nevertheless, this study raises the exciting possibility that defining different metabolic requirements among various memory $\mathrm{T}$ cells could provide a novel means for fine-tuning the regionalization of immune responses.

\section{J Michael Stolley ${ }^{1}$, David Masopust ${ }^{1}$}

${ }^{1}$ Department of Microbiology and Immunology, Center for Immunology, University of Minnesota Medical School, Minneapolis, MN, USA Correspondence: David Masopust

E-mail: masopust@umn.edu

\section{References}

1 Sallusto F, Lenig D, Förster R, et al. Nature 1999; 401:708-712.

2 Mueller SN, Gebhardt T, Carbone FR, et al. Annu Rev Immunol 2013; 31:137-161.

3 Thome JJ, Farber DL. Trends Immunol 2015; 36:428-435

4 Pan Y, Tian T, Park CO, et al. Nature 2017; 543:252-256.

5 Chang CH, Pearce EL. Nat Immunol 2016; 17:364-368.

6 Buck MD, O’Sullivan D, Pearce EL. J Exp Med 2015; 212:1345-1360.

7 O'Sullivan D, van der Windt GJ, Huang SC, et al. Immunity 2014; 41:75-88.

8 Cui G, Staron MM, Gray SM, et al. Cell 2015; 161:750-761.

9 Schenkel JM, Fraser KA, Beura LK, et al. Science 2014; 346:98-101.

10 Glennie ND, Yeramilli VA, Beiting DP, et al. J Exp Med 2015; 212:1405-1414.

11 Schenkel JM, Fraser KA, Casey KA, et al. $J$ Immunol 2016; 196:3920-3926.

12 Mackay LK, Wynne-Jones E, Freestone D, et al. Immunity 2015; 43:1101-1111. 